\title{
PET Measurement of Renal Glomerular Filtration Rate: Is There a Role in Nuclear Medicine?
}

\author{
M. Donald Blaufox \\ Department of Nuclear Medicine, Albert Einstein College of Medicine, Bronx, New York
}

$\mathbf{T}$ he article by Wakabayashi et al. in this issue of The Journal of Nuclear Medicine (1) describes the evaluation and potential use of ${ }^{18} \mathrm{~F}$-fluorodeoxy sorbitol as a potential PET agent, which can be used for the measurement of glomerular filtration rate (GFR). There is good reason to consider the possible use of a GFR agent in renal nuclear medicine. The standard of measurement of renal function used by the urologist and nephrologist is the GFR. Currently this is estimated using serum creatinine and creatinine estimated renal function, which is inaccurate and does not provide any information about individual renal function (2). Most of the attention of investigators in renal nuclear medicine in recent years has been directed toward agents that measure tubular function and effective renal plasma flow rather than GFR. This effort has been spearheaded by Dr. Andrew Taylor et al., who have introduced several compounds in recent years that are promising and have a high renal extraction (3). The advantage of an agent that is extracted to a high extent by the tubules for imaging with a SPECT camera

\section{See page 1625}

is significant. GFR agents are extracted only to the extent of about $20 \%$ or $25 \%$ of the amount in the blood, and therefore background activity is high during imaging from a variety of tissues in and around the kidneys. $\gamma$-camera evaluation of renal function with agents that are extracted by the tubules and that approximate the effective renal plasma flow is easier and background activity is reduced, thereby reducing the expected error in calculation of renal function. Several approaches have been advocated to correct for this background activity but none eliminate the problem $100 \%$, and none can be proven to be correct with the current methodology in place (4). There are several advantages to a PET agent in this situation. It is possible using PET to provide tomographic images of the kidney and virtually eliminate contributions to background from surrounding organs including the liver and spleen and major blood vessels. There still remains the problem of correcting for activity that is in the renal bloodstream and has not

Received Mar. 14, 2016; revision accepted Mar. 15, 2016.

For correspondence or reprints contact: M. Donald Blaufox, Albert Einstein College of Medicine, 165A Eastchester Rd., Bronx, NY 10461.

E-mail: mdonald.blaufox@einstein.yu.edu

Published online Apr. 21, 2016.

COPYRIGHT (C) 2016 by the Society of Nuclear Medicine and Molecular Imaging, Inc.

DOI: $10.2967 /$ jnumed.116.174607 actually been extracted by the kidney. In a highly vascular organ such as the kidney, this is a significant problem but one that could potentially be overcome once attention can be directed toward the intrarenal background alone and the problem of the overlying organs is eliminated.

Another reason for preferring tubular agents over GFR agents for evaluating the kidney with the $\gamma$-camera is that the high uptake and low background activity provide excellent images, which can be interpreted more accurately visually or by some automated system. GFR agents do not yield images with that quality on a camera imaging device. However, a GFR agent imaged on PET can provide images as free of background activity as renal plasma flow agents, which is shown by the images in the accompanying article (1). Unfortunately, although there is a potential advantage of using PET for its physical characteristics, the cost and radiation dose associated with PET/CT, compared with simple $\gamma$-camera imaging, at the present time may make that prohibitive. As PET and CT technology advance, the radiation problem can potentially be significantly reduced to present less of a problem (5). The other overwhelming difference is in the cost of performing a PET/CT study versus that of a simple $\gamma$-camera study (6). This problem too is likely to lessen as improved technology and efficiency reduce the cost of individual PET/CT studies.

The studies by Wakabayashi et al. are in rats. Although it is likely that their results can be replicated in humans, that still remains to be proven. Once that hurdle is overcome, there remains another: what conditions warrant a more complex and expensive study of the kidney to measure renal function? Szsabo et al. (7) reviewed the future direction of renal positron tomography in 2006, and their review provides some insight into potential future directions of renal studies using PET. A major application cited at that time was in the evaluation of renovascular hypertension. However, with time and improved medical therapy, the use of radionuclide methodology in patients with suspected renovascular hypertension has declined significantly. Another potential use cited was renal transplantation, but here again with new alternative methodologies nuclear medicine studies have declined. The applications, which at the present time appear to provide a potential role for PET, are in pediatrics and in those areas in which accurate renal functional assessment is much needed. Measurement of renal function in pediatrics can be difficult with noninvasive techniques because of changes in body mass and creatinine excretion, which make the use of creatinine clearance less reliable. Children also are more likely to have anatomic lesions such as ureteral pelvic obstruction or other anatomic abnormalities that interfere with renal function that require an anatomic assessment of the obstruction and of its functional significance with an agent, usually a 
diuretic, as well as some assessment of renal function in making decisions about potential removal of the kidney or repair. In these situations, the increased cost of PET along with its increased accuracy may warrant its use. Patients who have malignant lesions of the kidney and marginal renal function may benefit from more accurate combined anatomic functional assessment when the possibility of a partial versus a total nephrectomy is being considered. Studies in patients with drugs that affect renal function adversely may require PET especially in investigational situations in which subtle changes in renal function may be overlooked during the early phases of clinical trials. These are just a few of the potential uses for this technique. As our knowledge of the mechanism of disease and our ability to reverse disease processes that involve the kidney advance, it may well develop that PET/CT for renal functional measurement combined with imaging will become a preferred methodology in many of these situations.

The use of nuclear medicine for studies of the kidneys has declined significantly since the high level of excitement associated with its initial introduction into nuclear medicine. This is rather paradoxical, because one of the earliest and most exciting clinical uses of renal nuclear medicine was in the evaluation of individual renal function at a time when the studies were highly invasive and difficult to perform by other techniques. As methods for treating renovascular hypertension and transplant rejection have improved, interest in renal studies has declined along with the increased use of nonradiation techniques such as ultrasound. The challenge facing the renal nuclear medicine physician is to introduce methodology that is in step with the current state of the art in nephrology and urology and that will yield information that is not easily obtained by other noninvasive means. Studies such as the one reviewed here represent a promising step in the right direction.

\section{REFERENCES}

1. Wakabayashi H, Werner RA, Hayakawa N, et al. Initial preclinical evaluation of ${ }^{18} \mathrm{~F}$-fluorodeoxysorbitol PET as a novel functional renal imaging agent. $\mathrm{J} \mathrm{Nucl}$ Med. 2016;57:1625-1629.

2. Inker LA, Schmid CH, Tighiouart H, Eckfeldt JH, Feldman HI. Estimating glomerular filtration rate from serum creatinine and cystatin C. $N$ Engl J Med. 2012;367:20-29.

3. Klenc J, Lipowska M, Taylor AT. Synthesis and evaluation of ${ }^{99 \mathrm{~m}} \mathrm{Tc}(\mathrm{CO}) 3$ (FEDA): a new dual-purpose ${ }^{99 \mathrm{~m}} \mathrm{Tc} /{ }^{18} \mathrm{~F}$ renal imaging agent [abstract]. $\mathrm{J} \mathrm{Nucl}$ Med. 2015;56(5, suppl 3):654.

4. Blaufox MD. Editorial comments: renal background correction and measurement of split renal function-the challenge. Eur J Nucl Med Mol Imaging. 2016;43: 548-549.

5. Amis ES, Butler PF. ACR white paper on radiation dose in medicine: three years later. J Am Coll Radiol. 2010;7:865-870.

6. Delbeke D, Segall GM. Status of and trends in nuclear medicine in the United States. J Nucl Med. 2011;52:24S-28S.

7. Szabo Z, Xia J, Mathews WB, Brown PR. Future direction of renal positron emission tomography. Semin Nucl Med. 2006;36:36-50. 